\title{
On the spectral shift and the time delay of light in a Rindler accelerated frame
}

\author{
J. B. Formiga and C. Romero \\ Departamento de Física, Universidade Federal da Paraíba, \\ C.Postal 5008, 58051-970 João Pessoa, Pb, Brazil \\ E-mail: cromero@fisica.ufpb.br
}

March 27, 2018

\begin{abstract}
We discuss two effects predicted by the general theory of relativity in the context of Rindler accelerated observers: the gravitational spectral shift and the time delay of light. We show that these effects also appear in a Rindler frame in the absence of gravitational field, in accordance with the Einstein's equivalence principle.
\end{abstract}

\section{Introduction}

The concept of Rindler observers is usually taught in special relativity courses as an example of what is called an uniformly accelerated motion in Minkowski spacetime [1. An interesting property exhibited by this class of accelerated observers, which by definition move rectilinearly with constant proper acceleration, consists in the fact that if any two of these observers are connected with a solid rod, then no stresses appear in the rod, and this is useful to characterize the notion of a "rigid motion" in the context of special relativity 11. Apart from these pedagogical considerations, there has been a great deal of intererest in this kind of relativistic motion after the theoretical discovery, in the seventies, of the Unruh effect [3]. As is well known, this is a quantum effect and may be shortly described as follows. Let us suppose that in a certain inertial reference frame $S$ some quantum free field $\Phi$, defined in the entire Minkowski spacetime, is in the vacuum state, i.e. a state in which there is no particle. Consider now another frame $S^{\prime}$ in which the observers move with a constant proper acceleration with respect to $S$. What Unruh has showed is that these accelerated observers will detect not a vacuum state of the field $\Phi$, but instead a thermal distribution of particles depending on the proper acceleration of the observers [3]. This means that the concept of vacuum is relative and depends on the observer.

In spite of its quantum nature, it has been claimed recently that the Unruh effect has, in fact, a classical (non-quantum) origin due to different spacetime 
splittings adopted by the inertial and the accelerated observers 4, 5]. In the present paper we are interested in two (non-quantum) effects due to accelerated motion that, in principle, might be measured by Rindler observers: the spectralshift and the time delay of light. As we shall see, these two effects are also present in a Rindler frame in the absence of gravitation and, according to the principle of equivalence, they might well be interpreted by the accelerated observers as produced by real gravitational fields [6]. Thus the theoretical prediction of the existence of the two effects constitutes a simple example of Einstein's principle of equivalence.

This article is organized as follows. In Sec. I we give a brief review of the kinematics of Rindler observers. Sections II and III are devoted to the discussion of the spectral-shift and light time delay as seen by these accelerated observers. Our conclusions are summarized in Sec. IV.

\section{Rindler accelerated observers}

Let us consider the motion of an uniformly accelerated particle in Minkowski spacetime $M^{4}$ as viewed from an inertial reference frame $S$. The worldline of the particle is a timelike curve $\mathcal{C}$ which may be described by parametric equations $x^{\alpha}=x^{\alpha}(s)$, where $s$ is the arc length parameter of the curve. (Throughout this work we shall employ the usual notation $x^{\alpha}=\left(x^{0}=c t, x^{1}=x, x^{2}=\right.$ $\left.y, x^{3}=z\right)$, with $\alpha=0,1,2,3$, and consider Minkowski metric in the form $\left.\eta_{\alpha \beta}=\operatorname{diag}(+---)\right)$. An uniformly accelerated particle is defined, in special relativity, as the one whose proper acceleration (that is, the acceleration with respect to its instantaneous rest frame $S^{\prime}$ ) is constant 1]. The 4-velocity $u^{\alpha}$ and 4-acceleration $a^{\alpha}$ of the particle with respect to $S$ are definded as $u^{\alpha}=\frac{d x^{\alpha}}{d s}$ and $a^{\alpha}=\frac{d u^{\alpha}}{d s}$. Because we have chosen to parametrize $\mathcal{C}$ with $s$, the following conditions must hold

$$
\begin{gathered}
u^{\alpha} u_{\alpha}=\eta_{\alpha \beta} u^{\alpha} u^{\beta}=1, \\
a^{\alpha} u_{\alpha}=\eta_{\alpha \beta} a^{\alpha} u^{\beta}=0 .
\end{gathered}
$$

If $a$ denotes the magnitude of the three-dimensional acceleration vector of the particle with respect to $S^{\prime}$, then we have [2]

$$
a^{\alpha} a_{\alpha}=\eta_{\alpha \beta} a^{\alpha} a^{\beta}=-\frac{a^{2}}{c^{4}}
$$

For simplicity from henceforth we shall restrict ourselves to the one-dimensional motion in the $(1+1)$-dimensional Minkowski spacetime $M^{2}$. The conclusions, however, which will be drawn later from this simplification will naturally hold for the $(3+1)$-dimensional spacetime $M^{4}$. In the $(1+1)$-dimensional Minkowski spacetime we have $x^{\alpha}=\left(x^{0}=c t, x^{1}=x\right)$ and the equations (1), (2) and (3) read

$$
\left(u^{0}\right)^{2}-\left(u^{1}\right)^{2}=1
$$




$$
\begin{gathered}
a^{0} u^{0}-a^{1} u^{1}=0 \\
\left(a^{0}\right)^{2}-\left(a^{1}\right)^{2}=-\frac{a^{2}}{c^{4}}
\end{gathered}
$$

Since the Eq. (3) is the same in all frames of reference (recall that $a^{\alpha} a_{\alpha}$ is a Lorentz invariant quantity) the equations above may be used to find the motion of the accelerated particle with respect to the inertial frame $S$. With no loss of generality, let us consider the motion taking place in the positive direction of the $x$-axis. Then, from the equations (11), (2) and (3) we obtain the following system of ordinary differential equations

$$
\begin{aligned}
& \frac{d u^{0}}{d s}=\frac{a}{c^{2}} u^{1} \\
& \frac{d u^{1}}{d s}=\frac{a}{c^{2}} u^{0}
\end{aligned}
$$

We can choose the following initial conditions for the motion: $u^{0}(s=0)=1$ and $u^{1}(s=0)=0$, which implies that with respect to $S$, the particle is at rest $s=0$ []. Thus we have the solution

$$
\begin{aligned}
& u^{0}(s)=\frac{d x^{0}}{d s}=\cosh \left(\frac{a s}{c^{2}}\right) \\
& u^{1}(s)=\frac{d x^{1}}{d s}=\sinh \left(\frac{a s}{c^{2}}\right)
\end{aligned}
$$

Further integration of the above equations leads to

$$
\begin{aligned}
& x^{0}(s)=\frac{c^{2}}{a} \sinh \left(\frac{a s}{c^{2}}\right)+b^{0} \\
& x^{1}(s)=\frac{c^{2}}{a} \cosh \left(\frac{a s}{c^{2}}\right)+b^{1}
\end{aligned}
$$

where $b^{0}$ and $b^{1}$ are integration constants. For convenience we choose $b^{0}=0$ and $b^{1}=\frac{c^{2}}{a}$ as a second set of initial conditions (which means that when the accelerated particle is at $x=\frac{c^{2}}{a}$ its clock reads $\tau=t=0$, that is, both clocks the particle's and reference frame $S$ 's read the same time at $\tau=0$ ). Thus, the equations (77) become

$$
\begin{aligned}
& x^{0}(s)=\frac{c^{2}}{a} \sinh \left(\frac{a \tau}{c}\right) \\
& x^{1}(s)=\frac{c^{2}}{a} \cosh \left(\frac{a \tau}{c}\right)
\end{aligned}
$$

where we are now using the proper time $\tau$ as a parameter instead of $s$. [7 Let us briefly comment on the type of motion described by Eqs. (8) and (9) as seen by the observers of the inertial reference frame $S$. In a spacetime diagram the 
Figure 1: Hyperbolic motion in Minkowski spacetime representing the worldline of a particle that experiences a fixed acceleration with respect to its instantaneous rest frame.

worldline described by these equations corresponds to the hyperbola (see Fig.1)

$$
c^{2} t^{2}-x^{2}=\frac{c^{4}}{a^{2}}
$$

(This is why uniformly accelerated motion in Minkowski spacetime is customarily referred to as hyperbolic. [8]).

From the spacetime diagram represented in Fig.1 we see that when $t \rightarrow \infty$ the worldline of the particle approaches the lightcone of the event at the origin O of the coordinate system of $S$. A simple calculation shows that the velocity of the particle as seen from $S$ approaches $c$ when $t \rightarrow \infty$. Looking at the whole trajectory we see that the particle comes in decelerating from infinity to the origin $\mathrm{O}$ and then accelerates back towards $x=+\infty$. A further look at the diagram pictured in Fig.1 leads us to conclude that the right edges of the lightcone of $\mathrm{O}$ acts as an event horizon for the accelerated observer in the sense that only events that occur in the region $|c t|<x,(x>0)$, (known as the Rindler wedge) are accessible to the observer. At this point, let us note that it is possible to continuously fill the entire Rindler wedge with hyperbolic worldlines that correspond to accelerated observers with different values of the proper acceleration $a(0<a<\infty)$. ( Simply consider the equation (10) for various values of the parameter $a$.) It is usual to refer to this family of observers as 
Rindler observers. The frame constituted by Rindler observers (let us call it $S_{R}$ ) is the simplest example of a non-inertial frame in Minkowski spacetime, and due to its non-inertiality we expect, according to Einstein's principle of equivalence, that observers of $S_{R}$ can be led to think that they are in the presence of a "gravitational field". At this point let us recall that two effects that are typically associated with the presence of a real gravitational field are the spectral shift and the time delay of light. It is precisely in these two effects that we are interested in this paper, since according to Einstein's principle of equivalence they should also appear to accelerated observers, in particular to Rindler observers. For this purpose it is convenient to establish a coordinate system in which the accelerated observers are at rest. In other words, we want to have coordinates $(\tau, \xi)$ that give the proper time and the proper distance as measured by the accelerated observers.

A set of coordinates with the desired properties mentioned above is provided by the so-called Rindler coordinates. We now proceed to define these coordinates. First, let us mention that the particles associated with the Rindler frame may be regarded as constituting a rod "moving rigidly" (in a relativistic sense) in the $x$-direction. 1] Now out of the whole family of Rindler observers let us choose an observer $\mathcal{O}_{a}$ with a given proper acceleration $a$. Let us consider this observer as standing at one of the points of the rigid rod mentioned above. We may assign to the position of $\mathcal{O}_{a}$ in the rod the coordinate $\xi$, so then in $S_{R}$ a spacetime diagram the observer's worldline will correspond to the curve $\xi=0$. Let us consider another point $\mathcal{P}$ of the rod located at a proper distance $\xi$ with respect to $S_{R}$. The point $\mathcal{P}$, which is at rest (with respect to $S_{R}$ ), is accelerating together with the observer $\mathcal{O}_{a}$. Thus at an arbitrary time $\tau$ the proper coordinates of $\mathcal{O}_{a}$ and $\mathcal{P}$ are, respectively, $(\tau, 0)$ and $(\tau, \xi)$. Let $S^{\prime}$ be an inertial comoving frame at time $\tau$ with coordinates $\left(c t^{\prime}, x^{\prime}\right)$ in which the $x^{\prime}$-axis of $S^{\prime}$ coincides with $\mathcal{O}_{a}$ 's rigid rod and $x^{\prime}=0$ gives the position of $\mathcal{O}_{a}$. We also take $t^{\prime}=\tau$. In the coordinates of $S^{\prime}$. We now define in $S^{\prime}$ the "displacement vector" $l^{\prime \alpha}$ at time $\tau$ between $\mathcal{O}_{a}$ and $\mathcal{P}$ by $l^{\prime \alpha}=(\tau, \xi)-(\tau, 0)=(0, \xi)$. In the frame $S$ the components of the displacement vector may be obtained from the Lorentz transformation

$$
l^{\alpha}=\Lambda_{. \beta}^{\alpha} l^{\prime \beta}
$$

where $\Lambda_{-\beta}^{\alpha}=\left[\begin{array}{cc}\gamma & \beta \gamma \\ \beta \gamma & \gamma\end{array}\right], \gamma=\left(1-\frac{v^{2}}{c^{2}}\right)^{-1 / 2}, \beta=\frac{v}{c}$, and $v$ is the velocity $\frac{d x}{d t}$ of $\mathcal{O}_{a}$ with respect to $S$. Since $\gamma=u^{0}$ and $\beta \gamma=u^{1}$, we have from (6)

$$
\begin{aligned}
& l^{0}=x_{\mathcal{P}}^{0}-x_{\mathcal{O}}^{0}=u^{1} \xi=\xi \sinh \left(\frac{a s}{c^{2}}\right) \\
& l^{1}=x_{\mathcal{P}}^{1}-x_{\mathcal{O}}^{1}=u^{1} \xi=\xi \cosh \left(\frac{a s}{c^{2}}\right)
\end{aligned}
$$

Since the point $\mathcal{P}$ may be any point of the rigid rod we have just established the desired transformation between the coordinates of $S$ and that of $S_{R}$, which 
by virtue of 8 and $(9)$ yields

$$
\begin{aligned}
& x^{0}=\left(\frac{c^{2}+\xi a}{a}\right) \sinh \left(\frac{a \tau}{c}\right) \\
& x^{1}=\left(\frac{c^{2}+\xi a}{a}\right) \cosh \left(\frac{a \tau}{c}\right)
\end{aligned}
$$

The coordinates $(\tau, \xi)$ are defined in the intervals $-\infty<\tau<\infty$ and $-\frac{c^{2}}{a}<$ $\xi<\infty$ and it is easily seen that the line $\xi=-\frac{c^{2}}{a}$ is a horizon line for the accelerated observers (see Fig.1).

A simple calculation shows that the metric of Minkowski spacetime in the proper coordinates $(\xi, \tau)$ is given by

$$
d s^{2}=c^{2} d t^{2}-d x^{2}=\left(c+\frac{\xi a}{c}\right)^{2} d \tau^{2}-d \xi^{2}
$$

It is important to note that for Rindler observers the coordinate $\tau$ plays the role of a time coordinate and that the proper time $\Upsilon$ measured by an observer at $\xi=$ const is given by

$$
d \Upsilon=\left(1+\frac{\xi a}{c^{2}}\right) d \tau
$$

Of course the time coordinate and the proper time are the same for our observer $\mathcal{O}_{a}$ since by definition $\mathcal{O}_{a}$ has $\xi=0$. 9 ]

\section{Spectral-shift and time delay of light}

We now shall investigate two optical phenomena that in the context of the general theory of relativity are usually associated with the presence of gravitational fields. These are the spectral-shift and the time delay of light. Being essentially geometrical, these effects have an interpretation in terms of the bending of the spacetime due to the presence of matter. Nevertheless, they also may appear in flat Minkowski spacetime as a purely kinematical effect when we have accelerated observers. This should not surprise us since from the Einstein's principle of equivalence gravitational fields can be mimicked by non-inertial reference frames. Our aim in this section is to show how Rindler observers would detect and measure the spectral and time delay of light. As we shall see, this analysis can be easily carried out with the help of the Rindler coordinates $(\tau, \xi)$ defined in Sec. II. In the following we shall still be working in the (1+1)-Minkowski since the other two spatial dimensions are not relevant to the discussion.

Let us start with the spectral-shift. Consider the following experiment. Suppose that a ligth signal is sent by a Rindler observer $\mathcal{A}$ at a fixed point $\xi_{\mathcal{A}}$ at time $\tau_{\mathcal{A}}^{(1)}$ (with respect to the frame $S_{R}$ ), travels along a null geodesic and is received by another observer $\mathcal{B}$ a the fixed point $\xi_{\mathcal{B}}$ at time $\tau_{\mathcal{B}}^{(1)}$. Thus the signal passes from the event $E_{1}$ with coordinates $\left(\tau_{\mathcal{A}}^{(1)}, \xi_{\mathcal{A}}\right)$ to the event $R_{1}$ with coordinates $\left(\tau_{\mathcal{B}}^{(1)}, \xi_{\mathcal{B}}\right)$ (see Fig.2). 
Figure 2: The worldlines of two Rindler observers who perform an experiment to detect the spectral shift of light. The light signals travel along null geodesics from the observer $\mathcal{A}$ to the observer $\mathcal{B}$. 
Let $\lambda$ be an affine parameter along the null geodesic with $\lambda=\lambda_{1}$ at the event of the emission and $\lambda=\lambda_{2}$ at the event of reception. Since we have a null geodesic, it follows from (13) that

$$
\left(c+\frac{\xi a}{c}\right)^{2}\left(\frac{d \tau}{d \lambda}\right)^{2}-\left(\frac{d \xi}{d \lambda}\right)^{2}=0
$$

Because the signal travels from $\mathcal{A}$ to $\mathcal{B}$ and we are considering $\xi_{\mathcal{A}}<\xi_{\mathcal{B}}$ we have

$$
\frac{d \tau}{d \lambda}=\left(c+\frac{\xi a}{c}\right)^{-1} \frac{d \xi}{d \lambda}
$$

On integrating this equation we obtain

$$
\tau_{\mathcal{B}}^{(1)}-\tau_{\mathcal{A}}^{(1)}=c \int_{\xi_{\mathcal{A}}}^{\xi_{\mathcal{B}}} \frac{d \xi}{c^{2}+a \xi}
$$

Suppose now that a second light signal is emitted from $\mathcal{A}$ at time $\tau_{\mathcal{A}}^{(2)}$ (event $E_{2}$ ) and received by $\mathcal{B}$ at time $\tau_{\mathcal{B}}^{(2)}$ (event $R_{2}$ ). Since the integral on the right-hand side of (16) depends only on the fixed positions of the two observers we must have

$$
\tau_{\mathcal{B}}^{(2)}-\tau_{\mathcal{A}}^{(2)}=\tau_{\mathcal{B}}^{(1)}-\tau_{\mathcal{A}}^{(1)}
$$

which then implies

$$
\Delta t_{\mathcal{B}}=\tau_{\mathcal{B}}^{(2)}-\tau_{\mathcal{B}}^{(1)}=\tau_{\mathcal{A}}^{(2)}-\tau_{\mathcal{A}}^{(1)}=\Delta t_{\mathcal{A}}
$$

In other words, the coordinate time differences $\Delta t_{\mathcal{A}}$ and $\Delta t_{\mathcal{B}}$ between the emission and reception of the light signal as measured by $\mathcal{A}$ and $\mathcal{B}$ are the same. However, as we have mentioned earlier, the proper time measured by these observers depends on their position. From (14) the proper time differences $\Delta \Upsilon_{\mathcal{A}}$ and $\Delta \Upsilon_{\mathcal{B}}$ must be given by

$$
\begin{aligned}
\Delta \Upsilon_{\mathcal{A}} & =\left(1+\frac{\xi_{\mathcal{A}} a}{c^{2}}\right) \Delta t_{\mathcal{A}} \\
\Delta \Upsilon_{\mathcal{B}} & =\left(1+\frac{\xi_{\mathcal{B}} a}{c^{2}}\right) \Delta t_{\mathcal{B}}
\end{aligned}
$$

Since $\Delta t_{\mathcal{A}}=\Delta t_{\mathcal{B}}$, we have

$$
\frac{\Delta \Upsilon_{\mathcal{B}}}{\Delta \Upsilon_{\mathcal{A}}}=\frac{\left(1+\frac{\xi_{\mathcal{B}} a}{c^{2}}\right)}{\left(1+\frac{\xi_{\mathcal{A}} a}{c^{2}}\right)}
$$

This is the equation that leads to the spectral-shift formula. Indeed, suppose our light signal consists of $n$ waves of frequency $\nu_{\mathcal{A}}$, which are emitted in proper time $\Delta \Upsilon_{\mathcal{A}}$ from the observer $\mathcal{A}$. Then $n=\nu_{\mathcal{A}} \Delta \Upsilon_{\mathcal{A}}$. Observer $\mathcal{B}$ certainly 
receives $n$ waves, although the frequency and time duration of the wave train have changed. Clearly we also have $n=\nu_{\mathcal{B}} \Delta \Upsilon_{\mathcal{B}}$, which implies

$$
\nu_{\mathcal{B}}=\frac{\left(1+\frac{\xi_{\mathcal{A}} a}{c^{2}}\right)}{\left(1+\frac{\xi_{\mathcal{B}} a}{c^{2}}\right)} \nu_{\mathcal{A}}
$$

As we have considered $\xi_{\mathcal{A}}<\xi_{\mathcal{B}}$ Eq. (18) tells us that the frequency of light decreases as it leaves the observer $\mathcal{A}$ and when it is received at $\mathcal{B}$ we see a shift towards the red end of the spectrum. This interesting effect which, in principle, could be measured by accelerated observers, is the kinematical analogue of the gravitational red shift, which has been tested by experiment and been rather well verified. 10

Let us now turn our attention to the effect of the time delay of light. We know that in the presence of a gravitational field Einstein's general theory of relativity has shown that the travel time of light between two given points in space is greater than it would be in flat spacetime. This difference is called the light time delay and it has been very well verified experimentally. 11] We shall presently show that, as in the case of the spectral shift, an analogous effect may be measured by accelerated observers.

Consider, as previously, two observers $\mathcal{A}$ and $\mathcal{B}$ at the fixed points $\xi_{\mathcal{A}}$ and $\xi_{\mathcal{B}}$, respectively. Suppose that $\mathcal{A}$ sends a light signal towards $\mathcal{B}$ and that this signal is reflected back from $\mathcal{B}$ to $\mathcal{A}$ (See Fig.3).

We can now calculate the time lapse between the events of emission and reception of the signal. Again, because the signal travels along a null geodesic we have

$$
\frac{d \tau}{d \lambda}= \pm\left(c+\frac{\xi a}{c}\right)^{-1} \frac{d \xi}{d \lambda}
$$

Assuming now that $\xi_{\mathcal{A}}>\xi_{\mathcal{B}}$ the coordinate time $\Delta \tau$ for the whole trip will be given by

$$
\Delta \tau=-\int_{\xi_{\mathcal{A}}}^{\xi_{\mathcal{B}}}\left(c+\frac{\xi a}{c}\right)^{-1} d \xi+\int_{\xi_{\mathcal{B}}}^{\xi_{\mathcal{A}}}\left(c+\frac{\xi a}{c}\right)^{-1} d \xi=2 \int_{\xi_{\mathcal{B}}}^{\xi_{\mathcal{A}}}\left(c+\frac{\xi a}{c}\right)^{-1} d \xi
$$

The proper time lapse between the emission and reception of the light signal as recorded by the clock of the observer $\mathcal{A}$ will be given, according to Eq. (14), by

$$
\Delta \Upsilon=2\left(1+\frac{\xi_{\mathcal{A}} a}{c^{2}}\right) \int_{\xi_{\mathcal{B}}}^{\xi_{\mathcal{A}}}\left(c+\frac{\xi a}{c}\right)^{-1} d \xi=2 \frac{c}{a}\left(1+\frac{\xi_{\mathcal{A}} a}{c^{2}}\right) \ln \frac{c^{2}+a \xi_{\mathcal{A}}}{c^{2}+a \xi_{\mathcal{B}}}
$$

On the other hand because for the Rindler's observers the distance between the two observers $\mathcal{A}$ and $\mathcal{B}$ is $\Delta l=\xi_{\mathcal{A}}-\xi_{B}$ they would expect a round-trip time of

$$
\Delta \bar{\Upsilon}=2 \frac{\xi_{\mathcal{A}}-\xi_{\mathcal{B}}}{c}
$$

It can be shown that $\Delta \bar{\Upsilon}<\Delta \Upsilon$ (see Appendix) and this constitutes the time delay of light. The difference between $\Delta \bar{\Upsilon}$ and $\Delta \Upsilon$ is caused exclusively by the 
Figure 3: Light signal sent by the observer $\mathcal{A}$ towards the observer $\mathcal{B}$ and reflected back from $\mathcal{B}$ to $\mathcal{A}$. The measure of the time lapse between the events of emission and reception of the signal reveals the existence of time delay of light. 
acceleration of the Rindler's observers, that is, it wouldn't occur in a inertial reference frame. What we have here is a purely kinematical effect, which in a way reproduces the situation that appears when a real gravitational field is present.

\section{Final remarks}

We can give an idea of the order of magnitude of the redshift predicted by Eq. (18) by considering the observers $\mathcal{A}$ and $\mathcal{B}$ as corresponding to the worldlines $\xi_{\mathcal{A}}=0$ and $\xi_{\mathcal{B}}=10^{3} \mathrm{~m}$. For an acceleration $a=10 \mathrm{~m} / \mathrm{s}^{2}$ a simple calculation leads to $\frac{\nu_{B}-\nu_{A}}{\nu_{A}}=\frac{\Delta \nu}{\nu} \cong-10^{-12}$. It seems that in principle this predicted value is perfectly measurable with current measurement instruments. For comparison note that the gravitational red shift measured by Pound and Rebka experiment using a vertical distance of $22.5 \mathrm{~m}$ was $\frac{\Delta \nu}{\nu}=-(5.13 \pm 0.51) \times 10^{-15}$. [10].

We can also compute numerically the time delay of light for a configuration of observers such that $\xi_{\mathcal{B}}=0, \xi_{\mathcal{A}}=10^{11} \mathrm{~m}, a=10 \mathrm{~m} / \mathrm{s}^{2}$. In this case we obtain $\Delta \Upsilon-\Delta \bar{\Upsilon} \cong 3.7 \times 10^{-4} s$. We should note that the gravitational time delay, measured by Shapiro, between the transmission of radar pulses towards (Venus or Mercury) and the detection of the echos is of the order of $\Delta \Upsilon-\Delta \bar{\Upsilon} \cong$ $10^{-4} s$. 11] Let us remark that the distance involved in the measurement of the gravitational time delay effect is of the order of $10^{11} \mathrm{~m}$.

We conclude this section with the following general comment. It is well known that the equivalence principle, which establishes an equivalence between gravitational fields and accelerated observers, played a very important role in Einstein's formulation of the general theory of relativity. Indeed, it was this powerful physical principle that led him to predict the bending of light by a gravitatinal field and ultimately to the idea of geometrization of gravitation. 12] By considering a class of accelerated observers, namely Rindler observers, we have given a simple example of the validity and power of the principle when applied to two classical effects: the spectral shift and the time delay of light.

\section{Acknowledgement}

C. Romero would like to thank CNPq-FAPESQ (PRONEX) for financial support.

\section{Appendix}

In this appendix we show that $\Delta \bar{\Upsilon}<\Delta \Upsilon$ for arbitrary values of the coordinates $\xi_{\mathcal{A}}, \xi_{\mathcal{B}}$, provided that $\xi_{\mathcal{A}}>\xi_{\mathcal{B}}$. With this aim let us define the function $\mathcal{D}\left(\xi_{\mathcal{A}}, \xi_{\mathcal{B}}\right)$ as

$$
\mathcal{D}\left(\xi_{\mathcal{A}}, \xi_{\mathcal{B}}\right)=2 \frac{\xi_{\mathcal{A}}-\xi_{\mathcal{B}}}{c}-2 \frac{c}{a}\left(1+\frac{\xi_{\mathcal{A}} a}{c^{2}}\right) \ln \frac{c^{2}+a \xi_{\mathcal{A}}}{c^{2}+a \xi_{\mathcal{B}}}
$$


For an arbitrary fixed value of $\xi_{\mathcal{B}}$ let us examine the behaviour of this function when we vary $\xi_{\mathcal{A}}$. For this purpose take the derivative of $\mathcal{D}\left(\xi_{\mathcal{A}}, \xi_{\mathcal{B}}\right)$ with respect to $\xi_{\mathcal{A}}$. We have then

$$
\frac{\partial \mathcal{D}\left(\xi_{\mathcal{A}}, \xi_{\mathcal{B}}\right)}{\partial \xi_{\mathcal{A}}}=-\frac{2}{c} \ln \frac{c^{2}+a \xi_{\mathcal{A}}}{c^{2}+a \xi_{\mathcal{B}}}
$$

As $\xi_{\mathcal{A}}>\xi_{\mathcal{B}}$ then $\frac{\partial \mathcal{D}\left(\xi_{\mathcal{A}}, \xi_{\mathcal{B}}\right)}{\partial \xi_{\mathcal{A}}}$ is negative for all values of $\xi_{\mathcal{A}}, \xi_{\mathcal{B}}$. Now, for $\xi_{\mathcal{A}}=\xi_{\mathcal{B}}$ we have $\mathcal{D}\left(\xi_{\mathcal{B}}, \xi_{\mathcal{B}}\right)=0$. Therefore, we conclude that the function $\mathcal{D}\left(\xi_{\mathcal{A}}, \xi_{\mathcal{B}}\right)$ is negative for any value of the coordinates $\xi_{\mathcal{A}}, \xi_{\mathcal{B}}\left(\xi_{\mathcal{A}}>\xi_{\mathcal{B}}\right)$; hence the inequality $\Delta \bar{\Upsilon}<\Delta \Upsilon$ holds.

\subsection{Caption for figures:}

Fig.1 Hyperbolic motion in Minkowski spacetime representing the worldline of a particle that experiences a fixed acceleration with respect to its instantaneous rest frame.

Fig. 2 The worldlines of two Rindler observers who perform an experiment to detect the spectral shift of light. The light signals travel along null geodesics from the observer $\mathcal{A}$ to the observer $\mathcal{B}$.

Fig. 3 Light signal sent by the observer $\mathcal{A}$ towards the observer $\mathcal{B}$ and reflected back from $\mathcal{B}$ to $\mathcal{A}$. The measure of the time lapse between the events of emission and reception of the signal reveals the existence of time delay of light.

\section{References}

[1] See, for instance, W. Rindler, Essential Relativity (Springer-Verlag, New York, 1977, 2nd edition), Chap. 2

[2] Because in rest frame $S^{\prime}$ the velocity of particle $V^{\prime}=0$ the components of the 4 -acceleration in this frame are $a^{\prime \alpha}=\left(0,0, a / c^{2}, 0\right)$. As the $a^{\prime \alpha} a_{\alpha}^{\prime}$ is a relativistic invariant we have $a^{\prime \alpha} a_{\alpha}^{\prime}=a^{\alpha} a_{\alpha}=-\frac{a^{2}}{c^{4}}$.

[3] W. G. Unruh, Phys. Rev. D 14, 870 (1976).

[4] M. Pauri and M. Vallisneri, Found. Phys. Lett. 13, 401 (2000), gr-qc 9903052 v2.

[5] M. Lachièze-Rey, Astronomy and Astrophysics, 376, 17 (2001).

[6] A very discussion of the equivalence principle may be found in, J. Norton, "What was Einstein's Principle of Equivalence?", in Einstein and the History of General Relativity, D. Howard and J. Stachel (eds), Birkhäuser, Boston, 1989. 
[7] According to clock hypothesis of the theory of relativity, the proper time elapsed in the rest frame of particle between any two given events $A$ and $B$ may be calculated from the formula $\tau=\frac{1}{c} \int_{A}^{B} d s$, where the integration is performed along the path of the the particle. See, for instance, R. D'Inverno, Introducing Einstein's Relativity (Oxford, New York, 1992), Chap. 3.

[8] Curves corresponding to hyperbolic motion in Minkowski spacetime may be viewed geometrically as a special case of torsionless curves with constant curvature in a context where torsion and curvature are defined in a Lorentz invariant way. We refer the interested reader to J. B. Formiga and C. Romero, "On the differential geometry of timelike curves in Minkowski spacetime", Am. J. Phys. (2006) (to appear), gr-qc 0601002

[9] This gives the observer $\mathcal{O}_{a}$ a somewhat distinguished status in the building up of the Rindler coordinates. This point has been discussed recently by Silva and Dahia, who propose a "more democratic way" of defining a coordinate system for Rindler observers. See P.J. F. Silva and F. Dahia, "Space sections relative to a non-inertial frame in Minkowski spacetime" ( 2006), Proceedings of the XXIII Brazilian North-East Meeting on Physics.

[10] The gravitation redshift was first verified by the well known Pound-Rebka experiment carried out in the Jefferson Physics Laboratory at Harvard. The historical reference is R. V. Pound and G. A. Rebka, Phys. Rev. Lett. 4, 337-341 (1960).

[11] I. I. Shapiro, Phys. Rev. Lett. 13, 789-791 (1964). For recent results see B. Bertotti, L. Iess and P. Tortora, Nature, 425, 374 (2003).

[12] A. Einstein, "Über den Einfluss des Schwerkraft auf die Ausbreitung des Lichtes", Annalen der Physik 35, 898-908 (1911). Translated as "On the influence of Gravitation on the Propagation of Light", in H. Lorentz, The Principle of Relativity (Dover, New York, 1952). 